\title{
Influence of different chemotherapy regimens on the expression of ER and Her-2.
}

\author{
Yingchun Xu, Chaoyang Xu*, Liwei Meng, Shan Hu \\ Department of Breast and Thyroid Surgery, Shaoxing People's Hospital, Zhejiang University, Shaoxing City, PR China
}

\begin{abstract}
Objective: To investigate the influence of different chemotherapy regimens on the expression of Estrogen Receptor (ER) and Her-2.

Methods: Clinical pathology and the treatment of 78 patients with breast cancer were analyzed retrospectively, in which 39 were treated with Cyclophosphamide, Doxorubicin, Fluorouracil (CAF) regimen (group A), and the other 39 were treated with Docetaxel, Doxorubicin, Cyclophosphamide (TAC) Taxotere, regimen (group B). All of them were treated with chemotherapy for 4 cycles. The protein expressions of ER and Her-2 were compared before and after the therapy. The protein expressions of ER and Her-2 in different chemotherapy cycles and groups of patients were analyzed. The protein expressions of $\mathrm{ER}$ and Her-2 in different clinical stages were recorded.

Results: (1) After the neoadjuvant chemotherapy for 78 patients, the protein expression of ER decreased from $(1.53 \pm 0.16)$ to $(1.29 \pm 0.12), t=10.60, P=0.00$; the protein expression of Her- 2 decreased from $(1.32$ $\pm 0.07)$ to $(1.13 \pm 0.03), t=22.03, P=0.00$. (2) In this study, there were 40 patients with chemotherapy cycles less than three weeks and 38 patients equal or more than three weeks. Comparing the protein expression of ER in patients with chemotherapy cycles equal or more than three weeks with that less than three weeks, which was respectively $(1.41 \pm 0.18)$ and $(1.35 \pm 0.16), t=1.56, P=0.12$; comparing the protein expression of Her-2 in patients with chemotherapy cycles equal or more than three weeks with that less than three weeks, which was respectively $(1.27 \pm 0.05)$ and $(1.29 \pm 0.05), t=1.77, P=0.08$. (3) After chemotherapy, the average protein expression of $E R$ was $(1.27 \pm 0.14)$ in group $A$ and $(1.34 \pm 0.18)$ in group $B$. Comparing $A$ with $B, t=1.92, P=0.06$; the average protein expression of Her-2 was $(1.31 \pm$ $0.07)$ in group $A$ and $(1.30 \pm 0.09)$ in group $B$. Comparing $A$ with $B, t=1.73 P=0.09$. (4) Of the 78 patients, 36 were in stage I to II and 42 were in stage III to IV; comparing the average protein expression of ER of patients in stage I to II with that in stage III to IV, which was respectively $(1.29 \pm 0.12)$ and $(1.35 \pm 0.18)$, $t=1.70, P=0.09$; comparing the average protein expression of Her-2 of patients in stage $I$ to $I I$ with that in stage III to IV, which was $(1.15 \pm 0.07)$ and $(1.18 \pm 0.08)$ respectively, $t=1.75, P=0.08$.

Conclusion: The protein expressions of ER and Her-2 changed in breast cancer after chemotherapy. The protein expression of Her-2 in patients with longer chemotherapy cycles decreased when compared with that in patients with shorter chemotherapy cycles, while the expression of ER increased after chemotherapy. This may be attributed to the biological differences within the tumor tissue or the limited samples.
\end{abstract}

\section{Introduction}

Influenced by many factors such as environment and diet, the number of people with breast cancer is increasing and there is a trend that patients are younger and younger. Thus the physical and mental health of women is seriously threatened. Early diagnosis and timely treatment are the keys to improving the cure rate of breast cancer and reduce its lethality. Hence domestic and overseas medical researchers are focusing on how to achieve early detection and evaluate the therapy effect [1]. Neoadjuvant chemotherapy, which is mainly for patients without distant metastasis of breast cancer, is carried out before local chemotherapy. According to the relevant research [2], 24 $\mathrm{h}$ after the excision of primary lesion for breast cancer, the concentration of growth factor in peripheral circulation significantly increased, and the residual tumor cells could grow rapidly. However, residual tumor cells can be inhibited by preoperative cytotoxic therapy. Neoadjuvant chemotherapy can help prevent the micrometastatic lesion of peripheral tumors at early stages, inhibit the excision of primary lesion from stimulating the growth of residual lesion. At the same time, micrometastatic lesion can be excised by preoperative chemotherapy to avoid the emergence of drug-resistant cell lines and increase the survival rate. There is Estrogen Receptors (ER) in the mammary gland of human. When 
mammary epithelial cells turn cancerous, ER will partially or completely disappear. If ER can still be expressed in breast tumor tissues, it indicates tumor cells are still regulated by endocrine and endocrine therapy can be continued. So it has directive significance for clinical treatment and prognostic evaluation [3]. Her-2 is an important molecular marker of tumors. With its over-expression as a sign of malignant tumors, Her-2 is significant for prognostic evaluation and clinical treatment of breast cancer patients [4]. In this research, different chemotherapy regimens for breast cancer and the corresponding expressions of ER and Her-2 are studied to direct clinical treatment and improve prognosis of patients. The main content of this paper is as follows.

\section{Material and Method}

\section{Material}

Clinical pathology and treatment of 78 patients with breast cancer from April 2012 to March 2014 were analyzed retrospectively, in which 39 were treated with CAF regimen (group A), and the other 39 were treated with TAC regimen (group B). All of them were treated with chemotherapy for 4 cycles. All patients had no history of malignant tumors or benign breast diseases, and had tumor lesions that could be measured. After examinations such as B ultrasound, tumor size was detected to be more than $2 \mathrm{~cm}$. Cases that obtained complete remission or with progressed disease, as well as those with mental disease, other malignant tumors, allergic constitution, distant metastasis of breast cancer and no residual tumor cell after neoadjuvant chemotherapy, were excluded. TNM staging of tumor: 50 patients in stage II and 28 in stage III; all patients were women with single tumor; patients were aged from 39 to 72 , with an average age of (49.7 \pm 4.2$) ; 40$ were postmenopausal women and other 38 were premenopausal. After comparison of age and tumor staging of the two groups, $\mathrm{P}>0.05$ is comparable.

The concomitant administration of anthracyclines within the TAC regimen has been evaluated in several settings. In the NSABP B-38 there was no difference in Disease-Free Survival (DFS) and Overall Survival (OS) between six cycles of TAC with four cycles of dose-dense (dd) Doxorubicin and Cyclophosphamide (AC) followed by four cycles of $\mathrm{dd}$ paclitaxel while the toxicity profile was different, for instance grade three to four toxicities for TAC and dose dense chemotherapy were $9 \%$ versus $3 \%$ for febrile neutropenia, $<1 \%$ versus $7 \%$ for sensory neuropathy, and $7 \%$ versus $2 \%$ for diarrhoea. BCIRG 005 showed no difference in outcome between six cycles of TAC and four cycles of AC followed by four cycles of docetaxel every three weeks $(\mathrm{q} 3 \mathrm{w})$. Also here, TAC was associated with more febrile neutropenia and thrombocytopenia, while with the sequential regimen, more neuropathy, nail changes and myalgia were reported. On the contrary, NSABP-30 showed that four cycles of TAC was associated with worse DFS compared to four cycles of AC followed by four cycles of docetaxel every three weeks. Before G-CSF was administered routinely with the TAC regimen, febrile neutropenia (with occasional toxic deaths) was a major limitation for that regimen, but with Granulocyte-Colony Stimulating Factor, (G-CSF) and/or antibiotics prophylaxis, the febrile neutropenia rate seems clinically acceptable.

\section{Observation Index}

Preoperative (neoadjuvant) chemotherapy using doxorubicin and cyclophosphamide reduces the tumor size in patients with locally advanced disease enabling surgical resection. Recently, neoadjuvant chemotherapy is seen as a choice to patients with operable tumors who want breast conservation therapy. Though several clinical trials were conducted, no clear conclusion was drawn for disease-free survival or overall survival in the patients receiving preoperative therapy as opposed to those receiving postoperative chemotherapy.

Few investigations were carried out to study the effect of neoadjuvant chemotherapy on the hormone receptor status of primary breast carcinomas. No substantial differences were found in the expressions of ER, PR, and HER2 from diagnostic core biopsies before chemotherapy and final resection specimens after neoadjuvant therapy in thirty patients. Adams et al studied forty patients and found an increase in the proportion of tumors with HER2 overexpression following neoadjuvant chemotherapy but no change in hormone receptor status. As neoadjuvant chemotherapy is being practiced and due to the importance of clinical utility of Immunohistochemical (IHC) markers, any alteration in the IHC profile of breast carcinomas from chemotherapeutic agents affects the post-surgical utility of hormone-directed or HER2-specific therapy.

The protein expressions of ER and Her-2 were compared before and after the chemotherapy. The protein expressions of ER and Her-2 in different chemotherapy cycles and groups of patients were analyzed. The protein expressions of ER and Her-2 in different clinical stages were recorded. Specimens undergoing biopsy by Mammotome before chemotherapy and those excised after the therapy were collected. After fixed with $10 \%$ paraformaldehyde, specimens were paraffin embedded, cut into $4 \mu \mathrm{m}$-thick sections and then deparaffinized and hydrated. Endogenous enzyme was inactivated by ready-to-use $3 \% \mathrm{H}_{2} \mathrm{O}_{2}$ at room temperature for $10 \mathrm{~min}$; after antigen retrieval by citrate buffer under high temperature and high pressure, specimens were rinsed with distilled water and PBS in sequence and blocked by $5 \%$ bovine serum albumin blocking buffer at room temperature for $20 \mathrm{~min}$. Excess fluid was siphoned off. Corresponding first antibody was dropped into specimens (kept at $37^{\circ} \mathrm{C}$ for 1 hour or $4^{\circ} \mathrm{C}$ overnight and dipped in PBS for 3 times, 2 min for each time); then the second antibody was added into specimens (kept at $37^{\circ} \mathrm{C}$ for 30 min, and rinsed with PBS). Finally, specimens were colored by $\mathrm{DAB}$ for $10 \sim 15 \mathrm{~min}$, and rinsed with running water, until positive expression of specific parts was observed under the microscope. Specimens were counterstained with hematoxylin, dehydrated and sealed with neutral gum. $0.1 \mathrm{~mol} / \mathrm{L}$ PBS was used to replace the first antibody in negative control group, and sections tested positive were used in positive control group. All 
reagents were provided by Fuzhou Maixin Biotech. Co., Ltd. and ERER mouse anti-human polyclonal antibody was provided by Wuhan Boster Biological Technology Co., Ltd.

\section{Result}

\section{The protein expressions of ER and Her-2 before and after the chemotherapy}

After the neoadjuvant chemotherapy for 78 patients, the protein expression of ER decreased from $(1.53 \pm 0.16)$ to $(1.29$ $\pm 0.12), \mathrm{t}=10.60, \mathrm{P}=0.00$; the protein expression of Her-2 decreased from $(1.32 \pm 0.07)$ to $(1.13 \pm 0.03), \mathrm{t}=22.03, \mathrm{P}=0.00$.

\section{The protein expressions of Her-2 and ER in different chemotherapy cycles}

In this study, there were 40 patients with chemotherapy cycles less than three weeks and 38 patients equal or more than three weeks. Comparing the protein expression of ER in patients with chemotherapy cycles equal or more than three weeks with that less than three weeks, which was respectively $(1.41 \pm$ $0.18)$ and $(1.35 \pm 0.16), \mathrm{t}=1.56, \mathrm{P}=0.12$; comparing the protein expression of Her-2 in patients with chemotherapy cycles equal or more than three weeks with that less than three weeks, which was respectively $(1.27 \pm 0.05)$ and $(1.29 \pm 0.05), t=1.77$, $\mathrm{P}=0.08$.

\section{The protein expressions of ER and Her-2 in different chemotherapy regimens}

After chemotherapy, the average protein expression of ER was $(1.27 \pm 0.14)$ in group $\mathrm{A}$ and $(1.34 \pm 0.18)$ in group $\mathrm{B}$. Comparing A with $\mathrm{B}, \mathrm{t}=1.92, \mathrm{P}=0.06$; the average protein expression of Her-2 was (1.31 \pm 0.07$)$ in group $\mathrm{A}$ and $(1.30 \pm$ 0.09 ) in group $\mathrm{B}$. Comparing A with $\mathrm{B}, \mathrm{t}=1.73, \mathrm{P}=0.09$.

\section{The protein expressions of ER and Her-2 in different clinical stages}

Of the 78 patients, 36 were in stage I to II and 42 were in stage III to IV; comparing the average protein expression of ER in patients in stage I to II with that in stage III to IV, which was respectively $(1.29 \pm 0.12)$ and $(1.35 \pm 0.18), \mathrm{t}=1.70, \mathrm{P}=0.09$; comparing the average protein expression of Her-2 in patients in stage I to II with that in stage III to IV, which was $(1.15 \pm$ $0.07)$ and $(1.18 \pm 0.08)$ respectively, $\mathrm{t}=1.75, \mathrm{P}=0.08$.

\section{Discussion}

Breast cancer is a systemic disease, for there may be micrometastatic lesion in the early stage and the metastatic lesion can proliferate and grow faster in a short time when the primary lesion is excised. Therefore, the breast cancer is treated from operation of controllinglocal breast tissue and lymph gland to comprehensive therapy, making neoadjuvant chemotherapy a trend for treating breast cancer. Breast cancer is hormone-dependent, whose growth depends on estrogen and progesterone. The expression of ER can be used for clinical evaluation of endocrine therapy [5].

The result show that the protein expression of ER in patients decreased after chemotherapy, which is consistent with relevant literature [6]. Transcriptional reactions in all patients were significant during chemotherapy. After chemotherapy, genes in patients changed in different degrees and there was a significant genome difference between cases that were improved and those not. Another study [7] shows that hormone receptor-negative patients are more sensitive to chemotherapy, so changes of hormone receptor are more likely to occur in patients greatly improved after chemotherapy. In other words, cancer cells with negative ER are easily killed to increase the protein expression of ER. It is, however, not in consistent with the results of this study, which may be caused by the sample or method adopted. In this study, Western Blot was used so the result might be affected by factors such as the efficiency of the antibody itself, for protein might dissolve or high molecular weight protein might be affected by ultrasound and so on. The decrease of protein expression of Her- 2 might be related to its sensitivity to anthracycline-based chemotherapy, so tumor cells with high expression of Her-2 were killed selectively [8].

In this study, comparing the protein expression of ER in patients with chemotherapy cycles equal or more than three weeks with that less than three weeks, $\mathrm{P}=0.12$; comparing the protein expression of Her-2 in patients with chemotherapy cycles equal or more than three weeks with that less than three weeks, $\mathrm{P}=0.08$. This may be due to the limited efficacy of anthracycline-based chemotherapy on tumor cells with high expression of Her-2, which is in a dose-dependent manner. Besides, patients with negative hormone receptor are more sensitive to chemotherapy. After chemotherapy, comparing protein expressions of ER and Her-2 in group A with those in group $\mathrm{B}, \mathrm{P}=0.06, \mathrm{P}=0.09$ correspondingly. Comparing the protein expressions of ER and Her-2 of patients in stage I to II with those in stage III to IV, $\mathrm{P}=0.09, \mathrm{P}=0.08$ correspondingly. It may be explained that when compared with patients in early clinical stages, those in late stages had tumors with longer growth time and severer deterioration, resulting in high protein expression of gene related to breast cancer [9]. There is ER in normal mammary gland. However, when mammary epithelial cells turn cancerous, ER will partially or completely disappear. So if ER can still be expressed in breast tumor tissues, it indicates tumor cells are still regulated by endocrine and the regulative function of normal cells has not been completely destroyed, suggesting a better prognosis of patients [10]. Clinical treatment failure of patients with breast cancer is due to the metastasis and relapse of tumor, making it important to adopt an appropriate treatment regimen [11-12].

In conclusion, the protein expression of ER decreased after chemotherapy but the difference was not obvious. So in this study, it cannot be inferred that the expression change was related to chemotherapy. The protein expression of Her-2 decreased after chemotherapy, which may be caused by the high proliferating activity of tumor cell and its sensitivity to chemotherapeutics. Because the number of samples is limited 
in this study, further study is needed to confirm the effects of various chemotherapy regimens onprotein expressions of Her-2 and ER with a large number of samples.

\section{References}

1. Jing D, Xiaoli Z, Lina J. Expression of estrogen receptor, progesterone receptor and Her-2 in breast cancer and its clinical significance. Chinese J Gerontol 2014; 34: 5405-5406.

2. Hong X, Fengxia Z, Kaiping Y. Correlation of the serum hormone estradiol and progesterone levels and estrogen and progesterone receptors with the disease associated with breast. Chinese J Clin 2013; 7: 5275-5278.

3. Ping H, Anzhi W, Yingchun Y. Relationship of Her-2 gene amplification with estrogen receptor, progesterone receptor and lymph node metastasis in invasive breast cancer. Maternal Child Health Care China 2013; 28: 3501-3504.

4. Jun T, Juanjuan Z, Jun Z. The expression of Cyclin D1 and its correlation with estrogen receptor and progesterone receptor in different molecular subtypes of breast cancer. Chinese J Exp Surgery 2014; 31: 968-970.

5. Yufeng $\mathrm{Y}$, Jinhai $\mathrm{T}$, Rong $\mathrm{M}$. The expression of drug resistance protein and its correlation with estrogen receptor, progesterone receptor and Her-2 in different molecular subtypes of breast cancer. Chinese J Oncol 2014; 36: 771-772.

6. Gang B. Efficacy of breast cancer neoadjuvant chemotherapy and the expression change of estrogen receptor and progesterone receptor. J Guiyang Med College 2015; 40: 737-740.

7. Wentao Y, Hong B. Guideline for immunohistochemical testing of estrogen receptor and progesterone receptor in breast cancer. Chinese J Pathol 2015; 35: 237-239.
8. Haiyan M, Juanjuan L, Chunjian Q. Observation on the effect of autologous dendritic cell vaccine on patients with estrogen receptor/progestin receptor double-negative breast cancer. Chinese Clin Oncol 2013; 18: 1005-1008.

9. Yan Z, Enli W, Hui L. The abnormal expression of MiRNAs in serum of patients with breast cancer and its potential role as a diagnostic biomarker. Maternal Child Health Care China 2013; 28: 4865-4867.

10. Shuanggen L, Xiaofen Y, Qiumo L. Expression of proliferating cell nuclear antigen and its correlation with estrogen receptor, progesterone receptor and Her-2 in breast cancer for elderly women. Chinese J Gerontol 2015; 20: 5817-5818.

11. Jia Y, Guanqiao L, Xiaojie Z. Analysis on correlation between expression of estrogen receptor and progesterone receptor and clinicopathological factors of breast cancer and the significance of combined detection. Maternal Child Health Care China 2015; 30: 3968-3970.

12. Lei L, Xiru L, Jin Z. Association between the expression of IGF1R and estrogen receptor and efficacy of neoadjuvant chemotherapy in breast cancer. Chinese J Oncol 2015; 37: 833-836.

\section{*Correspondence to}

Chaoyang $\mathrm{Xu}$

Department of Breast and Thyroid Surgery

Shaoxing People's Hospital

Zhejiang University

PR China 